\title{
Giant intracavernous aneurysm presenting with isolated trigeminal neuralgia: a case report
}

\author{
Fadhilah Hani Ishak', Abdul Hanif Khan Yusof Khan ${ }^{1 *} \mathbb{0}$, Mohd Naim Mohd Yaakob², \\ Mohd Fandi Al Khafiz Kamis², Anna Misyail Abdul Rashid', Wei Chao Loh', Janudin Baharin', Azliza Ibrahim", \\ Liyana Najwa Inche Mat', Wan Aliaa Wan Sulaiman' ${ }^{1}$, Fan Kee Hoo ${ }^{1}$ and Hamidon Basri ${ }^{1}$
}

\begin{abstract}
Background: Trigeminal neuralgia (TN) commonly affects individuals aged more than 50 years and is mostly primary owing to trigeminal nerve neurovascular compression. We report a case of secondary TN attributed to a giant intracavernous aneurysm compressing the trigeminal nerve.

Case presentation: A 74-year-old female presented with classic TN symptoms, which were initially refractory to medical treatment. Imaging including MRI brain, MR angiography, and cerebral angiogram revealed a giant intracavernous aneurysm measuring $1.7 \mathrm{~cm} \times 2.8 \mathrm{~cm}$, inducing mass effect on the left Meckel's cave. Aneurysm embolization was challenging due to the difficult cannulation of the distal portion of the aneurysm. The patient opted for conservative management with mild improvement of symptoms.

Conclusions: Intracavernous aneurysm rarely present with isolated trigeminal neuralgia. This case report emphasizes that the assessment of the intracranial vasculature should be considered as part of the diagnostic imaging work-up for patients presenting with TN
\end{abstract}

Keywords: Intracranial aneurysm, Trigeminal neuralgia, Embolization therapeutic, Elderly, Case report

\section{Background}

Trigeminal neuralgia ( $\mathrm{TN})$, also known as tic douloureux, is described as recurrent brief episodes of unilateral electric shock-like pains, abrupt in onset and termination, in the distribution of one or more divisions of the fifth cranial (trigeminal) nerve [1]. It is usually triggered by touching the skin lightly or other specific activities. The pain can be excruciating stabbing pain within less than $20 \mathrm{~s}$, often leaving patients with uncontrollable facial twitching [2].

This condition mainly affects individuals more than 50 years of age [1]. It is mostly primary and is classic TN due to the trigeminal nerve's compression without an apparent cause [1]. The major contributive factor in

\footnotetext{
*Correspondence: ahanifkhan@upm.edu.my

${ }^{1}$ Department of Neurology, Faculty of Medicine and Health Sciences,

University Putra Malaysia, 43400 Serdang, Selangor, Malaysia

Full list of author information is available at the end of the article
}

classic TNs is compression of the trigeminal nerve root or near the dorsal root entry zone by a blood vessel, usually an artery [3]. Although TN in younger patients can be a sign of secondary disease, this is not always the case. Possible causes of secondary TN include multiple sclerosis, cerebellopontine angle tumours, and arteriovenous malformations [1]. It can also be mimicked by a dental abscess, nasopharyngeal tumours, sinusitis, and temporomandibular joint arthritis [4].

Cavernous carotid aneurysms, classified under secondary TN, are asymptomatic in $18 \%$ of people with this condition [5]. They mainly present with diplopia and pain usually portrayed as retro-orbital pain or headache [5]. While isolated third nerve palsy and isolated sixth nerve palsy are also commonly reported, isolated trigeminal dysesthesia associated with headache is rarely found [5]. This case report reports a rare case of a giant intracavernous carotid aneurysm causing isolated TN. 


\section{Case presentation}

A 74-year-old female with a background of hypertension and a history of burr hole surgery for intracranial bleeding in 1998 has been complaining of left-sided facial pain for 2 years since 2018. She described the pain as intermittent sharp severe pain mainly triggered by touch, particularly over the left maxillary area region, and occasionally was associated with numbness. There was no neurological deficit, and other cranial nerve palsies were not observed. She was diagnosed with TN by her primary care doctors and was intermittently prescribed analgesia to no avail. She was then subsequently referred to our center for further evaluation.

Blood investigations performed for this patient were unremarkable. She proceeded with a magnetic resonance imaging (MRI), which revealed a giant intracavernous left internal carotid artery (ICA) aneurysm. The left ICA aneurysm was seen impinging on the left trigeminal nerve, as shown in Figs. 1 and 2.

She was treated with oral pregabalin $50 \mathrm{mg}$ once daily initially as she decided for conservative management. Pain initially improved with pregabalin for 3 months; however, the pain subsequently relapsed with no pain recovery, and the patient was not keen to further increase the pregabalin dose due to the feeling of dizziness. The

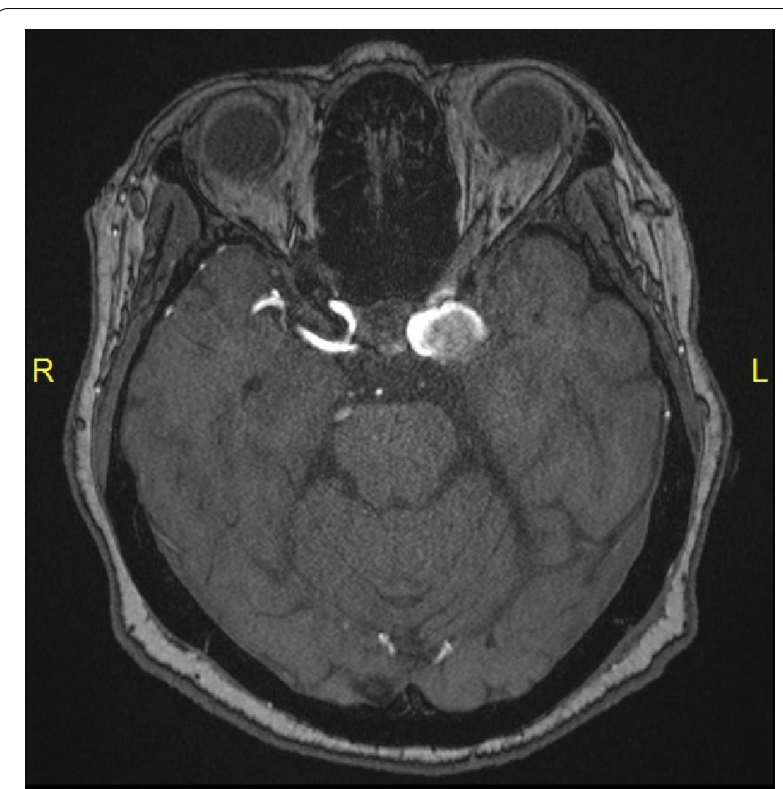

Fig. 2 MRA shows arterialization of the lesion suggestive of the left internal carotid aneurysm

pain worsened and became constant; therefore, the patient and family decided to discuss intervention during the discussion with the interventional radiologist.

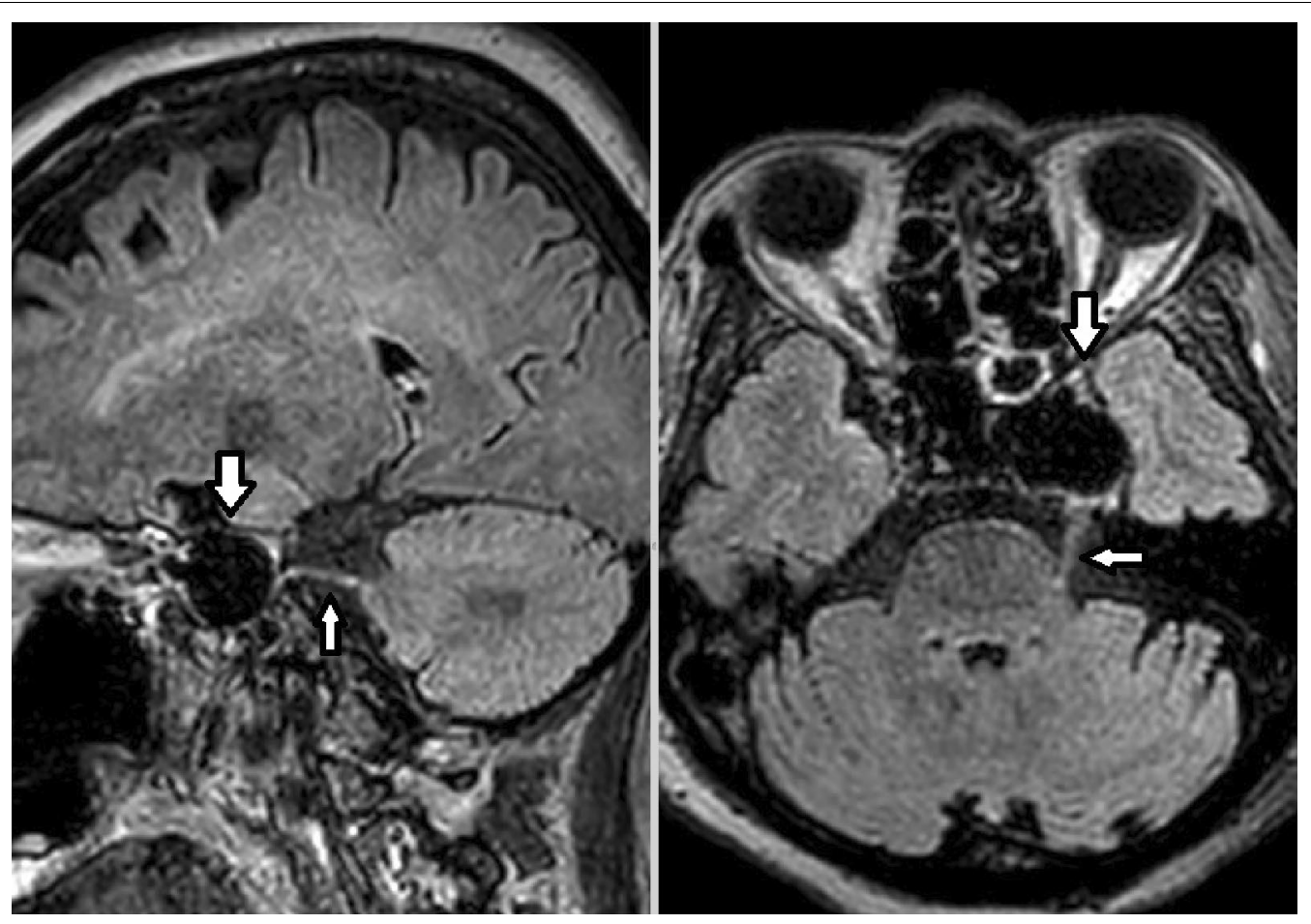

Fig. 1 3D-FLAIR in axial and sagittal showed a giant aneurysm (hypointense, big arrow) located at the left cavernous sinus inducing mass effect to the left Meckel's cave. The hyperintense left trigeminal nerve (small arrow) is impinged by the aneurysm within the left Meckel's cave, causing trigeminal neuralgia 
She was planned for a cerebral angiogram and flowdiverter stent and coiling. The carotid angiogram revealed a giant intracavernous aneurysm of the left ICA, causing a mass effect on Meckel's cave, which was similar in size compared to previous imaging, as shown in Fig. 3 . The aneurysm was not extending to the arachnoid space. Unfortunately, embolization failed due to the difficult cannulation of the distal part of the aneurysm. We also attempted to flip the micro-guidewire within the aneurysm to support the microcatheter; however, it was unsuccessful. The patient required admission post-procedure for close observation as she developed transient hypotension and hypothermia.

She made an uneventful recovery post-intervention and, we opted for medical therapy with a continuation dose of pregabalin $50 \mathrm{mg}$ daily with slow titration to $75 \mathrm{mg}$ daily as the patient was not keen on surgical intervention. We also added a low dose of gabapentin $300 \mathrm{mg}$ at night with careful explanation to family members to watch for dizziness and fall prevention. She was still having intermittent pain during follow-up, but tolerable with current medical treatment and not keen on further invasive intervention.

\section{Discussion}

Primary TN is more commonly seen in older patients. Neurovascular compression in the elderly is likely related to arteriosclerosis, given the increasing prevalence of arteriosclerosis with age. Arteriosclerosis gradually changes the shape and conformation of the vessel, potentially causing neurovascular compression [6]. This relationship may also explain the higher incidence of arterial neurovascular compression in older TN patients. Our case was different, whereby a giant intracavernous carotid aneurysm was found impinging on the trigeminal nerve causing pain over the maxillary distribution of the left trigeminal nerve.

Intracranial aneurysms causing facial pain may have multiple locations, including supraclinoid ICA, anterior ICA, vertebrobasilar, posterior-cerebral (PCA), cavernous ICA, and trigeminal artery [7, 8]. Intracavernous carotid aneurysms are commonly complicated by cranial nerve palsies, particularly oculomotor nerve, trochlear nerve, ophthalmic and maxillary branches of the trigeminal nerve, and the abducens nerve, which transverse the cavernous sinus [9]. Despite that, they rarely cause isolated trigeminal nerve palsy but have been found to cause isolated abducens, oculomotor or trochlear nerve palsy $[5,10]$. In our case, the cranial nerve involved is solely the trigeminal nerve causing constant sharp facial pain ipsilateral to the giant left ICA intracavernous aneurysm.

Diagnosis of TN warrants imaging in all cases, especially if clinical suspicions are towards the presence of compressive (vascular on non-vascular) or inflammatory lesions of the trigeminal nerve. Imaging is particularly crucial if the pain is refractory to initial medical management or if the presentation is atypical [1]. The presence of the giant left ICA aneurysm in our patient explains why the facial pain became unresponsive towards pregabalin. Other possible secondary causes of TN include arteriovenous malformations, cerebellopontine angle tumours, nasopharyngeal tumours, and multiple sclerosis [1]. These conditions can be excluded utilizing imaging, particularly MRI.

Several challenges were encountered while managing this case. Improvement of symptoms shown towards initial medical treatment with pregabalin was seen progressed to refractory after a few months. Failure of medical treatment leads to endovascular treatment, which would reduce the risk of rupture and the formation of

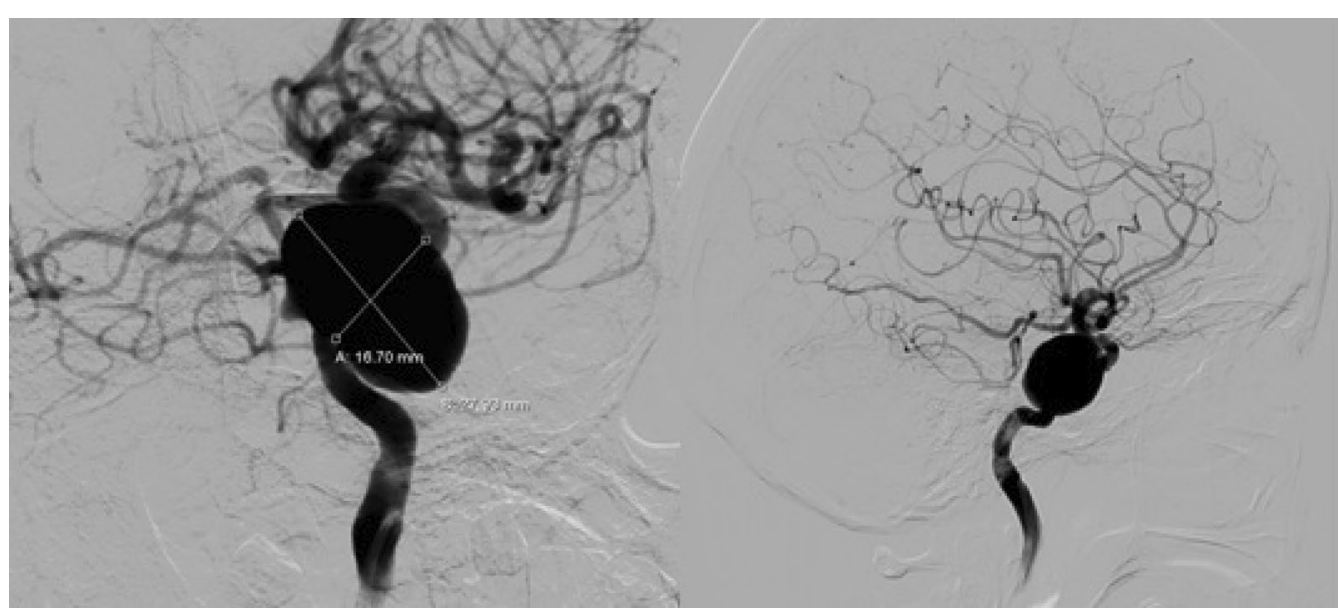

Fig. 3 Left internal carotid artery (ICA). Conventional angiogram showed fusiform dilatation of the left ICA measuring $1.7 \mathrm{~cm} \times 2.8 \mathrm{~cm}$ 
carotid-cavernous fistula and, at the same time, relieve the facial pain. Despite effective cannulation utilizing microwire, the key obstacle in our case that led to procedure failure was the difficulty in getting the microcatheter (for stent deployment) into the internal carotid artery distal to the aneurysm. Attempts to flip micro-guidewire within the aneurysm to provide support for the microcatheter were likewise unsuccessful.

Although pregabalin showed a reduction in the early treatment period, the first-line treatment in classic TNs (neurovascular compression) is pharmacological therapy with carbamazepine or oxcarbazepine [11]. The same drugs have also been suggested in secondary TNs, although placebo-controlled trials are lacking [11]. Other alternatives include baclofen, lamotrigine, pimozide, gabapentin, pregabalin, levetiracetam, botulinum toxin Type A and phenytoin [11]. The endovascular approach is the preferred method for the treatment of intracavernous carotid aneurysms. The introduction of flow-diverter stents has been shown safe and effective with very low mortality $(0-0.44 \%)$ and morbidity rates $(2.3-3.1 \%)$ with nearly $100 \%$ occlusion rates [12]. Another strategy that can be used is ICA occlusion with or without bypass utilizing balloon test occlusion (BTO) test before the procedure to assess tolerability of ICA occlusion [12].

\section{Conclusions}

An intracavernous aneurysm can rarely present with isolated trigeminal neuralgia. This case report emphasizes that the assessment of the intracranial vasculature should be considered as part of the diagnostic imaging work-up for patients presenting with $\mathrm{TN}$.

\section{Abbreviations}

ICA: Internal carotid artery; ICU: Intensive care unit; MRI: Magnetic resonance imaging; MRA: Magnetic resonance angiography; PCA: Posterior cerebral artery; TN: Trigeminal neuralgia.

\section{Acknowledgements}

We would like to thank Mrs F for her consent in publishing this case report.

\section{Authors' contributions}

FHI, AHKYK, AMAR, LNIM, and HB diagnosed the patient and collected clinical data. FHI, AHKYK, JB, WCL, Al, AMAR and LNIM carried out the literature search, review, and manuscript preparation. MNMY and MFAKK collected imaging data and preparing review on radiology. FKH, WAWS, and $\mathrm{HB}$ review the manuscript and approved the final draft. All authors read and approved the final manuscript.

\section{Funding}

Not applicable.

\section{Availability of data and materials}

The data sets supporting the conclusion of this article are included within the article.

\section{Declarations}

Ethics approval and consent to participate

We confirm that ethical clearance was not required for publication of this case report.

\section{Consent for publication}

Written informed consent to publish this information was obtained from study participant.

Competing interests

Authors confirm that they have no competing interests.

\section{Author details}

'Department of Neurology, Faculty of Medicine and Health Sciences, University Putra Malaysia, 43400 Serdang, Selangor, Malaysia. ${ }^{2}$ Department of Radiology, Faculty of Medicine and Health Sciences, University Putra Malaysia, 43400 Serdang, Selangor, Malaysia.

Received: 9 February 2021 Accepted: 6 August 2021

Published online: 26 August 2021

\section{References}

1. Shankar Kikkeri N, Nagalli S. Trigeminal neuralgia. Treasure Island: StatPearls Publishing; 2020.

2. Trigeminal Neuralgia_Causes, Symptoms and Treatments. 2020. https:// www.aans.org/Patients/Neurosurgical-Conditionsand-Treatments/Trige minal-Neuralgia. Accessed 27 Aug 2020.

3. Nurmikko TJ, Eldridge PR. Trigeminal neuralgia—pathophysiology, diagnosis and current treatment. Br J Anaesth. 2001;87:117-32. https://doi. org/10.1093/bja/87.1.117.

4. Trotter MI, Choksey MS. Facial pain with intracranial aneurysm. J R Soc Med. 2000:93:479-80. https://doi.org/10.1177/014107680009300908.

5. Stiebel-Kalish H, Kalish Y, Bar-On RH, Setton A, Niimi Y, Berenstein A, Kupersmith MJ. Presentation, natural history, and management of carotid cavernous aneurysms. Neurosurgery. 2005;57:850-7. https://doi.org/10. 1227/01.neu.0000179922.48165.42.

6. Chakravarthi PS, Ghanta R, Kattimani V. Microvascular decompression treatment for trigeminal neuralgia. J Craniofac Surg. 2011;22:894-8. https://doi.org/10.1097/SCS.0b013e31821a07b7.

7. Dzierzanowski J, Słoniewski P. Trigeminal neuralgia caused by aneurysm of the posterior cerebral artery: a case description and the analysis of anatomical variety of vascular complex in the root entry zone of Trigeminal nerve. Folia Morphol. 2014;73:224-8. https://doi.org/10.5603/FM.2014. 0033 .

8. Sughrue ME, Saloner D, Rayz VL, Lawton MT. Giant intracranial aneurysms: evolution of management in a contemporary surgical series. Neurosurgery. 2011:69:1261-70. https://doi.org/10.1227/NEU.0b013e31822bb8a6.

9. Brown A, Jolliff H, Poe D, Weinstock M. Intracavernous internal carotid artery aneurysm presenting as acute diplopia: a case report. Clin Pract Cases Emerg Med. 2020;4:362. https://doi.org/10.5811/cpcem.2020.3. 45266.

10. Goldenberg-Cohen N, Curry C, Miller NR, Tamargo RJ, Murphy KP. Long term visual and neurological prognosis in patients with treated and untreated cavernous sinus aneurysms. J Neurol Neurosurg Psychiatry. 2004;75:863-7. https://doi.org/10.1136/jnnp.2003.020917.

11. Obermann M. Treatment options in trigeminal neuralgia. Ther Adv Neurol Disord. 2010;3:107-15. https://doi.org/10.1177/1756285609359317.

12 Sattur M, Saway BF, Lena J, Spiotta A. Endovascular management of a giant skull base cavernous aneurysm: parent artery occlusion is still a relevant strategy. Cureus. 2021. https://doi.org/10.7759/cureus.13643.

\section{Publisher's Note}

Springer Nature remains neutral with regard to jurisdictional claims in published maps and institutional affiliations. 